\title{
Associação da alta frequência, laser de baixa potência e microcorrentes no tratamento da lesão por pressão
}

\author{
Association of high frequency, low-power laser and \\ microcurrent in the treatment of pressure injury
}

\author{
Claudia Maria Schuh,, ${ }^{1}$ Kimberly Araujo Alves, ${ }^{1}$ Letícia Wollmann, ${ }^{1}$ Patrícia Ribeiro Rodrigues, ${ }^{1}$ \\ Taís Oliveira Araújo, ${ }^{1}$ Ana Cristina Sudbrack' \\ Universidade de Santa Cruz do Sul (UNISC), Santa Cruz do Sul, RS, Brasil.
}

Recebido em: 25/10/2016 / Aceito em: 20/02/2017 / Publicado em: 31/03/2017 cms@unisc.br

\section{RESUMO}

A fisioterapia conta hoje com diversos recursos para o tratamento da lesão por pressão, com bons resultados, tais como a alta frequência, o laser e a microcorrente. Objetivo: demonstrar os efeitos da associação de três recursos da eletroterapia, a alta frequência, a microcorrente e o laser no tratamento do reparo tecidual em lesão por pressão. Método: trata-se de um relato de caso de um indivíduo do sexo masculino, 25 anos, com lesão de pressão em estágio 4, devido ao longo período acamado ou em sedestação na cadeira de rodas devido ao acometimento de lesão medular. Inicialmente, foi avaliado para a coleta de dados demográficos, clínicos e o exame físico, em que foi observada a área da lesão, a presença de inflamação/infecção, áreas de endurecimento e isquemia. A ferida apresentava-se, inicialmente, coberta com necrose e, após o desbridamento, com perda total da pele, envolvendo dano do tecido subcutâneo que se estendia até a fáscia subjacente. No início das sessões realizadas, era feita a limpeza local da lesão com soro fisiológico e utilizado o gerador de alta frequência com eletrodo cogumelo pequeno, utilizando o método de aplicação direta, pelo tempo de 10 minutos, abordando toda a extensão da lesão. Alternadamente, foi utilizada a microcorrente, com frequência de $600 \mathrm{~Hz}$ com dois canais distribuídos em forma de cruz com $100 \mathrm{~Hz}$ de intensidade em cada um, durante 25 minutos; e o laser de baixa potência, com emissão de feixe contínuo, 660nm de comprimento de onda, aplicado no modo pontual com intensidade de 12 $\mathrm{J} \backslash \mathrm{cm}^{2}$ pelo tempo determinado pelo equipamento para cada ponto. Foram realizadas 15 sessões, 3 vezes por semana. Resultados: ao final do protocolo, foi possível perceber uma diminuição significativa do tamanho da lesão de pressão onde se observou uma redução na área de $81 \%$. Conclusão: a associação dos recursos eletroterapêuticos utilizados, alta frequência, laser e microcorrente, mostraram resultados satisfatórios no tratamento da lesão de pressão.

Palavras-chave: Cicatrização; Eletroterapia; Lesão por pressão.

\section{ABSTRACT}

Physiotherapy nowadays has many resources for the treatment of pressure injury, with good results, such as high frequency, laser and microcurrent. Aim: demonstrate the effects of association of three resources of electrotherapy: high-frequency, microcurrent and laser at treatment of tissue repair for pressure injury. Method: this is a case report of a male patient, 25 years with stage IV pressure injury due to long period bedridden or sitting position in a wheelchair due to spinal cord injury. Initially it was collect demographic data, clinical and physical examination where the area of the ulcer was observed, presence of inflammation/infection, hardening areas and ischemia. The wound was initially covered with necrosis and, after debridement, with total loss of skin involving damage of the subcutaneous tissue that extended to the underlying fascia. At the beginning of meetings local cleaning of the ulcer was made with saline and used the high-frequency generator with small mushroom electrode using the direct application method, 10-minute time, addressing all the extent of the ulcer. Alternately, the microcurrent was used, with a $600 \mathrm{~Hz}$ frequency with two channels distributed 
in a cross shape with an intensity of $100 \mathrm{~Hz}$ each for 25 minutes. The low-power laser with emission continuous beam, 660nm wavelength, applied in timely manner with an intensity of $12 \mathrm{~J} / \mathrm{cm}^{2}$ by the time determined by the equipment for each point. 15 sessions were performed, 3 times per week. Results: at the end of protocol it was possible to see a significant reduction in the size of pressure ulcer where there was a reduction in area of $81 \%$. Conclusion: the association of electrotherapeutic resources used (high frequency, laser and microcurrent) showed satisfactory results in the treatment of pressure ulcers.

Keywords: Wound Healing; Electric Stimulation Therapy; Pressure injury.

\section{INTRODUÇÃO}

Conforme a literatura, as lesões por pressão, também conhecidas por escaras, têm sua fisiologia descrita pelo aumento da intensidade de pressão em determinada área do corpo, causando um aumento secundário da pressão nos capilares sanguíneos, resultando em uma isquemia local, vindo a acarretar desarranjos no aporte de nutrientes, levando a uma disfunção metabólica tegumentar, muscular e óssea, causando assim uma necrose celular.

Neste sentido, entende-se que a lesão se origina nas áreas corporais que estão submetidas à pressão constante e prolongada. ${ }^{2}$ Segundo Smeltzer, ${ }^{3}$ diversas pesquisas suportam a teoria em que as lesões se desenvolvem sobre proeminências ósseas, sendo que a maior parte delas aparece em membros inferiores, com alta incidência em regiões sacrais, tuberosidades isquiáticas, calcâneo e trocânter maior do fêmur, devido à maior concentração do peso corporal, o que potencializa o aumento da pressão em relação à superfície.

Porém, existem situações associadas às condições crônico-degenerativas das lesões por pressão, como acidentes vasculares cerebrais, diabetes mellitus, infartos, hipertensão arterial, trauma e outras patologias que afetam especialmente os idosos. ${ }^{4}$

Alguns recursos têm mostrado bons resultados no que se refere ao tratamento destas afecções, como a microcorrente que se apresenta como uma corrente alternada que utiliza parâmetros de baixa frequência e intensidade na faixa dos microampéres. O plano de atuação da microcorrente pode ser superficial e profundo, podendo atingir nível muscular, e apresenta-se com imediata atuação no plano cutâneo e subcutâneo. ${ }^{5}$ O objetivo terapêutico da microcorrente em lesões cutâneas é organizar o fluxo de correntes, acelerar o processo de reparação tecidual, produzir efeito bactericida e diminuir a dor. ${ }^{6}$

A laserterapia também aparece como terapia de escolha no tratamento das lesões, pois se mostra efetiva na aceleração da cicatrização de feridas, sendo considerado um recurso importante e que exerce efeito positivo sobre o processo da lesão, diminuindo consideravelmente o tempo de cicatrização. O laser terapêutico de baixa potência estimula a atividade mitocondrial, causando um aumento da síntese de Trifosfato de adenosina (ATP) e em adição oferece um efeito de sinalização celular, promovendo uma proliferação celular e citoproteção, além de intensificar a reabsorção da fibrina, aumentar a quantidade de tecido de granulação e diminuir a liberação de mediadores inflamatórios, processo esse que, em conjunto com outros fatores, acelera o processo de reparação tecidual. ${ }^{7}$ Como principais efeitos fisiológicos, estão a ação antiinflamatória, neoangiogênese, proliferação epitelial e de fibroblastos, síntese e deposição de colágeno, revascularização e contração da ferida. ${ }^{8}$

Outro recurso que também tem sido descrito na literatura e que tem apresentado boa eficácia no tratamento desta injúria dérmica, é o gerador de alta frequência (AF), que opera com correntes alternadas (tensão elevada e baixa intensidade) e eletrodos de vidro, que contêm em seu interior vácuo (ar rarefeito) ou gás (neon). ${ }^{5}$ Aliado à geração de campo elétrico, o gerador de $\mathrm{AF}$ tem capacidade de produzir alterações fisiológicas com efeitos imediatos na prática clínica, provavelmente por sua ação bactericida, antisséptica e anti-inflamatória, as quais podem favorecer a reparação tecidual. ${ }^{9}$

Portanto, percebe-se a necessidade da utilização do gerador de alta frequência no início de cada intervenção a fim de promover a assepsia da ferida, já que se trata de tecido vulnerável à infecção, especialmente quando não existe no local serviço especializado para tal.

A utilização do laser de baixa potência tem sido apresentada, na literatura disponível, como um recurso eficaz com potencial de acelerar o processo de cicatrização, aumentar o tecido de granulação, contrair a ferida, diminuir o processo inflamatório e reduzir a dor, apresentando redução significativa no tamanho das lesões, quando comparadas às não tratadas, porém, ainda necessitando de um tempo considerável de tratamento. ${ }^{10,11}$

Em relação ao uso da microcorrente no reparo das lesões, o que se encontra descrito na literatura é de que foram realizadas de 30 a 60 sessões de uso isolado desta terapêutica para gerar um resultado satisfatório. 5,12

O objetivo do estudo foi demonstrar os efeitos da associação destes três recursos eletrotermofototerápicos no tratamento do reparo tecidual de lesão por pressão, buscando uma redução no tempo de tratamento necessário em relação aos já relatados, com a utilização dos recursos de forma isolada.

\section{MÉTODO}

Trata-se de um relato de caso realizado através da análise de um prontuário da clínica FisioUnisc da Universidade de Santa Cruz do Sul, localizada no município de Santa Cruz do Sul-RS. O caso tratado é de um paciente, do sexo masculino, 25 anos, com diagnóstico clínico de fratura completa de corpo vertebral (C5), o qual desenvolveu uma lesão na região sacral em estágio 4 , medindo $17 \mathrm{~cm}$ em sua largura e $13 \mathrm{~cm}$ em seu comprimento, totalizando $221 \mathrm{~cm}^{2}$ devido ao fato de permanecer por grandes períodos acamado ou em sedestação na cadeira de rodas. Quando chegou para a fisioterapia, realizava tratamento clínico, baseado em curativos simples (medidas gerais de higienização da lesão com soro fisiológico e oclusão com gaze). O referido paciente assinou um termo de consentimento utilizado na clínica Fisiounisc que autoriza o uso de seus dados para fins de pesquisa e de divulgação em publicações científicas.

Inicialmente, o paciente foi submetido a uma avaliação para coleta de dados demográficos e clínicos 
utilizando-se o Instrumento de Avaliação do Estado da Lesão de Pressão, proposto por Barbara Bates-Jensen ${ }^{13}$ e adaptado a este estudo, o qual avalia a localização, forma, tamanho, profundidade, contornos, tipo de tecido e exsudato, tecido de granulação e epitelização e também através da Classificação das Lesões por Pressão por Estágios, ${ }^{14}$ que representa a quantidade de perda ou destruição tecidual ocorrida. A avaliação ocorreu em três momentos (antes da intervenção, após 8 sessões e ao término do protocolo estabelecido, após 15 sessões).

Nas sessões realizadas, primeiramente a lesão era limpa com soro fisiológico e o protocolo para o tratamento foi constituído da seguinte forma: utilizando o gerador de alta frequência (Tone Derm, modelo Versatile AF9); o eletrodo eleito foi o cogumelo pequeno com método de aplicação direta no modo varredura, pelo tempo de 10 minutos, abordando toda a extensão da lesão, logo após foi utilizada a microcorrente de mesmo modelo e marca, com frequência de $600 \mathrm{~Hz}$ com dois canais distribuídos em forma de cruz com $100 \mathrm{~Hz}$ de intensidade em cada um; o tempo estabelecido foi de 25 minutos. Na sessão seguinte, novamente, após a limpeza prévia com soro fisiológico, era utilizada a alta frequência da mesma forma e após o laser (Ibramed, modelo Laserpulse), que foi utilizado com emissão de feixe contínuo, 660nm de comprimento de onda, no modo pontual com intensidade de $12 \mathrm{~J} \mathrm{~cm}^{2}$ pelo tempo determinado pelo equipamento para cada ponto. $E$ assim, foi utilizado, alternadamente, ao longo das sessões, um dia o AF mais a microcorrente e na sessão seguinte, o AF mais o laser. No total foram realizadas 15 sessões de fisioterapia, 3 vezes por semana durante 45 minutos.

\section{RESULTADOS E DISCUSSÃO}

Inicialmente, observou-se extensa área de necrose na região sacral e parte das regiões glúteas, apresentando borda de cor rosa esbranquiçada e, na região superior, a lesão estava aberta com exposição das estruturas ósseas da região sacral (Figura 1A).

Posteriormente, a extensa área de necrose foi submetida à desbridamento para a remoção dos tecidos necrosados limpando o leito da ferida, antes de iniciar o referido tratamento, possibilitando então as intervenções fisioterapêuticas (Figura 1B).

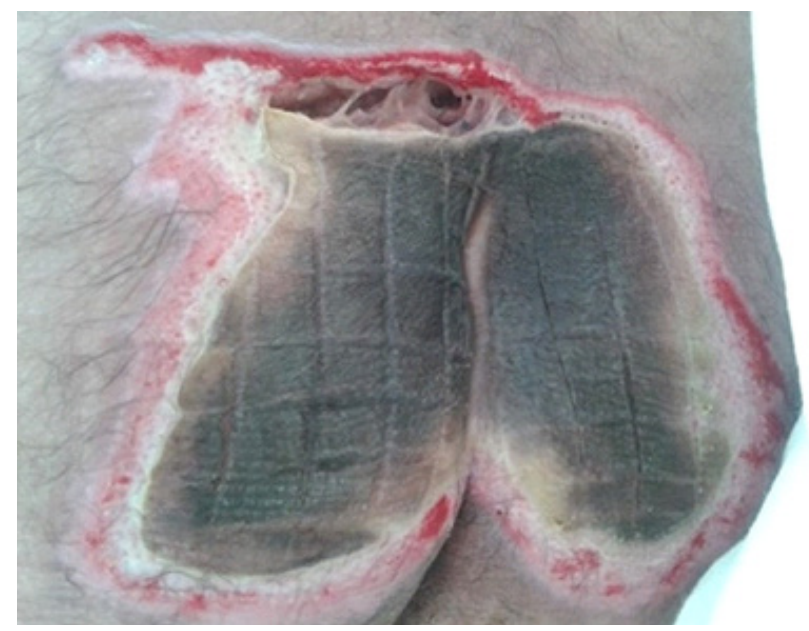

Figura $1 \mathrm{~A}$

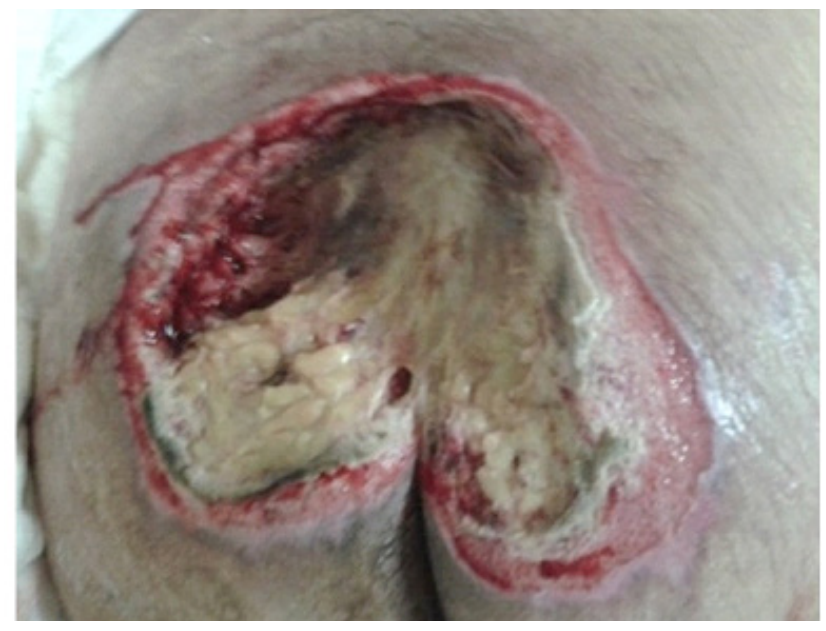

Figura 1B

Após 8 sessões de terapêutica já foi possível a percepção de neovascularização em toda área da lesão, ausência de necrose e de infecção e diminuição no tamanho da lesão que, em suas medidas iniciais apresentava $17 \mathrm{~cm} \mathrm{X} 13 \mathrm{~cm}, 221 \mathrm{~cm}^{2}$ para $14,5 \mathrm{~cm} \times 10 \mathrm{~cm}$, $145 \mathrm{~cm}^{2}$, apresentando uma redução de $34 \%$ (Figura 2A). Na décima quinta sessão é possível avaliar uma redução significativa do tamanho da escara, com as medidas de $7 \mathrm{~cm} \times 6 \mathrm{~cm}$ ou $42 \mathrm{~cm}^{2}$, demonstrando uma redução total de $81 \%$ da área, encontrando-se a feriada em estágio 3, onde observa-se perda da pele em sua espessura total sem nenhum tecido necrótico visível, cor da pele circundante ao tecido de cor rosa, com escasso exsudato. (Figura 2B).

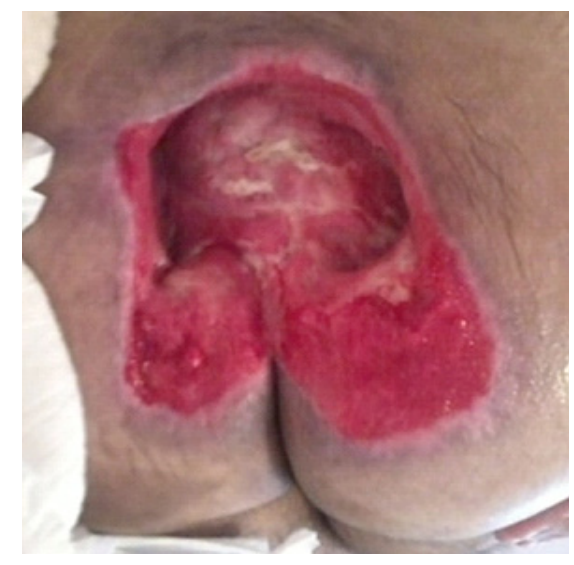

Figura 2A

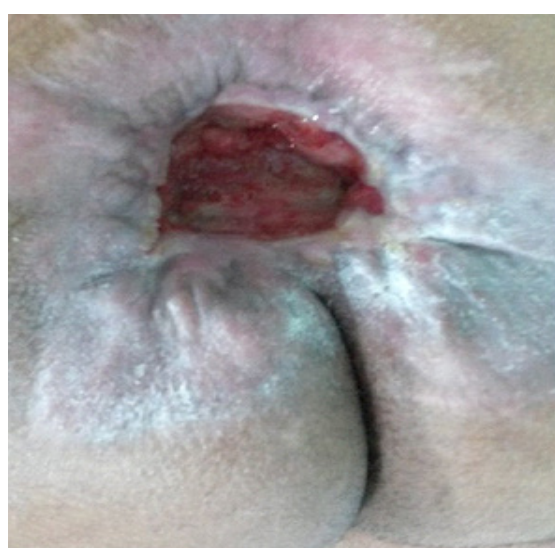

Figura 2B 
Como citado anteriormente, foi utilizado no protocolo de tratamento a alta frequência associada à microcorrente intercalada com o Laser, onde se observou uma redução no tamanho da área da lesão de $81 \% \mathrm{com}$ 15 aplicações. Borges ${ }^{5}$ cita o trabalho realizado em lesão de pressão, com o uso somente da microcorrente que apresentou uma redução de $60 \%$ do tamanho da lesão em 60 aplicações, evidenciando-se, assim, que a associação de recursos no nosso estudo mostrou-se mais efetiva.

Nos resultados do estudo de Alves ${ }^{15}$ foi mencionado que, após o uso da microcorrente, as lesões cutâneas apresentaram-se mais hiperemiadas, sugerindo o aumento do fluxo sanguíneo no local, e que as bordas da ferida estavam contraindo-se, demonstrando o tensionamento tecidual no sentido da cicatrização. As sessões de atendimento eram realizadas três vezes por semana, em dias alternados, totalizando 30 sessões; ao final das sessões a redução do tamanho da ferida foi de $92,2 \%$, sugerindo que a microcorrentes pode ser uma alternativa terapêutica no processo de reparo de feridas.

Medeiros $^{16}$ investigou os efeitos do uso do laser, da microcorrente e da terapia combinada (laser e microcorrente) no processo de reparo tecidual em lesões cutâneas. Tratou-se de um estudo quase experimental, cuja amostra foi composta por 9 participantes, divididos em três grupos de forma aleatória. Os atendimentos foram realizados 3 vezes por semana, durante 8 semanas. Todos os participantes que utilizaram laserterapia, microcorrente e terapia combinada demonstraram uma aceleração no processo de reparo tecidual com redução do tamanho das lesões cutâneas.

No nosso estudo utilizou-se o laser com a dose de $12 \mathrm{~J} \mathrm{~cm}^{2}$, Lopes, ${ }^{17}$ em seu estudo, utilizou a dose de $17 \mathrm{~J} \backslash \mathrm{cm}^{2}$ por 12 sessões, tanto no leito da lesão, quanto nas bordas e concluiu que o laser de baixa potência AIGalnP, $660 \mathrm{~nm}$, atuou com sucesso no processo de cicatrização das lesões de pressão, visto que, nesse caso, o resultado também foi satisfatório.

Em outro estudo, onde foi utilizado o laser de baixa potência no tratamento de lesões, os sujeitos da amostra foram submetidos à aplicação do laser com comprimento de onda de $658 \mathrm{~nm}$ e a dose escolhida de 4 joules $/ \mathrm{cm}^{2}$. Esse estudo constituiu-se de três casos clínicos, nos quais foi avaliado estágio inicial e final de cada lesão apresentada pelos sujeitos. O caso A apresentava lesões sacral, no qual foram realizadas quatro sessões de laserterapia. Observou-se 41,63\% de evolução. No caso $B$ foram realizadas 10 sessões de laserterapia nas lesões na região do sacro. A evolução obtida entre a avaliação inicial e $5^{\mathrm{a}}$ sessão foi de $60,78 \% .^{18}$

Quanto à utilização da alta frequência em todas as sessões foi possível observar, no nosso estudo, que a utilização da técnica promoveu resultados positivos, quanto à revascularização e a diminuição da área da lesão, o que vai ao encontro do estudo realizado por Korelo, ${ }^{19}$ onde pacientes com diagnóstico clínico de lesão por pressão grau 2 e 3, em que foram divididos em dois grupos: controle $(n=2)$ e alta frequência ( $n$ = 6), com aplicação diária durante dez dias, com 10 minutos de aplicação, sendo variável com o tamanho da lesão (1 minuto para cada $\mathrm{cm}^{2}$ ). O grupo no qual foi realizada aplicação da alta frequência com a técnica de faiscamento, amplitude a $80 \%$ e eletrodo do tipo bico, contornando toda a lesão e o seu interior, afastado do tecido o suficiente para evitar o contato e a contaminação, totalizou duas semanas de intervenção. Foi concluído com esse grupo que os resultados obtidos sugerem que a aplicação da alta frequência em pacientes com lesões por pressão proporciona melhora na cicatrização das lesões, bem como a diminuição da área de superfície da lesão.

Martins ${ }^{20}$ realizou um estudo analítico experimental in vitro com o objetivo de verificar o efeito bactericida da AF sobre a cultura de Staphylococcus aureus, sendo utilizada a técnica de faiscamento em quinze aplicações durante quinze minutos sobre as placas e demonstrou que, após 48 horas, 95\% das placas submetidas ao AF não tiveram crescimento de Staphylococcusaureus, assim, o gerador de alta frequência apresentou um efeito bactericida sobre esta cultura.

Higa $^{21}$ demonstrou em seu estudo in vitro, a ação fungicida da AF em cultura de cândida tropicalis, realizado com técnica de faiscamento, em dez aplicações por cinco minutos sobre as colônias, demonstrou que, após 48 horas $90 \%$ das placas submetidas ao $A F$, não tiveram crescimento de cândida; já, as placas que não receberam aplicação tiveram crescimento normal.

Com base nestes estudos e, com os resultados obtidos no tratamento do paciente de nosso estudo, é possível inferir que os efeitos bactericida e fungicida do gerador de alta frequência, já estão estabelecidos na literatura, porém, ainda existe uma carência de publicações de estudos em seres humanos, especialmente no que diz respeito ao tratamento da lesão por pressão para demonstrar a eficácia da AF no tratamento de reparo tecidual.

\section{CONSIDERACÕES FINAIS}

A realização desse trabalho permitiu mostrar que os resultados alcançados foram satisfatórios no tratamento da lesão de pressão com a associação dos três recursos terapêuticos utilizados: alta frequência, laser e microcorrente, especialmente no que diz respeito ao reduzido tempo de tratamento.

Foi possível comprovar a efetividade do tratamento quanto aos efeitos fisiológicos descritos, o que ocasionou uma melhora da cicatrização e expressiva diminuição da área da lesão.

\section{REFERÊNCIAS}

1. Guyton AC, Hall JE. Tratado de Fisiologia Médica. Rio de Janeiro: Elsevier 2006; $11^{\mathrm{a}}$ edição.

2. Irion G. Feridas Novas abordagens, manejo clínico e Atlas em cores. Rio de Janeiro: Guanabara Koogan, 2005; $2^{a}$ edição.

3. Smeltzer SC, Bare BG. Tratado de enfermagem médicocirúrgico. Rio de Janeiro: Guanabara Koogan, 2000.

4. Medeiros AF. Lesão por pressão em idosos hospitalizados: análise da prevalência e fatores de risco [dissertação]. Fortaleza-CE: Universidade Estadual do Ceará, 2006.

5. Borges FS. Dermato-Funcional Modalidades Terapêuticas nas disfunções Estéticas. São Paulo: Phorte, 2010; $2^{a}$ edição.

6. Caloy L. Necessidades da atuação da fisioterapia dermatofuncional em uma instituição de longa permanência de idosos 
[dissertação]. Porto Alegre-RS: Universidade Católica do Rio Grande do Sul, 2011.

7. Furieri FPM, Helena MU, Oliveira RR, Fagundes DS. Atuação Fisioterapêutica na lesão por pressão: uma revisão. Rev Cient Facul Edu Meio Amb 2015;(6)1:69-80.

8. Andrade FSSD, Clark RMO, Ferreira ML. Efeitos da Laserterapia de baixa potência na cicatrização de feridas cutâneas. Rev Col Bras Cir 2014;41(2):129-33.

9. Oliveira LMN. Through the use of ozone in high frequency device in the treatment of pressure ulcers. Rev Bras Cienc Saúde 2011;9(30):41-6. doi: 10.2149/tmh.2015-12

10. Palagi S, Severo IM, Menegon DB, Lucena AF. Laser therapy in pressure ulcers: evaluation by the Pressure Ulcer Scale for Healing and Nursing Outcomes Classification. Rev Esc Enferm USP 2015;49(5):820-826 doi: 10.1590/S0080623420150000500017

11. Taradaj J, Halski T, Kucharzewski M, Urbanek T, Halska U, Kucio C, Litscher G. Effect of laser irradiation at diferente wavelengths $(940,808$ and $658 \mathrm{~nm})$ on pressute ulcer healing: results from a clinical study. Evidence-Based Complementary and Alternative Medicine. Volume 2013 Article ID 960240. doi:10.1155/2013/960240

12. Martelli A, Theodoro V, Zanibon VE, Freitas, BA, Patre GM, Melo KM, Andrade TAM, Santos GMT.The microcurrent in the wound healing process: literature review Arch Health Invest 2016;5(3):134-139. doi: 10.21270/archi.v5i3.1316

13. Bates-Jensen B, Sussman C. Tools to measure wound healing. In Sussman C, Bates-Jensen B, editors. Wound Care, a Collaborative Practice Manual for Health Professionals, 4 ed. Baltimore (US): Lippincott Williams and Wilkins; 2012. p. 131-72.
14. Moraes JT, Borges EL, Lisboa CR, et al. Enferm. Cent. O. Min 2016;6(2):2292-2306. doi: 10.19175/recom.v6i2.1423

15. Alves GPB, Leal CT, Carneiro RF, Alves R, Resende TR. Utilização da microcorrente no processo de cicatrização de feridas em paciente pós-traumático: Estudo de caso. In: Congresso Brasileiro de Fisioterapia Dermato Funcional, Recife - Pernambuco: Brafidef. 2012; 1-6.

16. Medeiros ML, Pinto JMD, Silva ACJS, Silva GM, Figueirêdo LSM. O uso do laser e da microcorrente em lesões cutâneas [resumo]. 2011; 1.

17. Lopes LDF. Utilização de laser de $660 \mathrm{~nm}, 17 \mathrm{~J} / \mathrm{cm} 2 \mathrm{em}$ lesões por pressão- Um relato de caso. Rev Neurocienc 2011;19(4):668-74.

18. Felice TD, Pinheiro RA, Menchlk EDS, Silva ACD, Souza LS, Caires CSA, Abel A, Bartmeyer CG, Oliveira JG, Assis TB, Silva LA, Lopes TF, Felippe LA, Pinheiro AR. Utilização do Laser de baixa potencia na cicatrização de feriadas 2009;3:42-52.

19. Korelo RIG, OliveiraJJJ, Souza RSA,Hullek RF,Fernandes LC. Gerador de alta frequência como recurso para tratamento de lesões por pressão: estudo piloto. Fisioter Mov 2013;26(4):71524. doi: 10.1590/S0103-51502013000400002

20. Martins A, Silva JT, Graciola L, Fréz AR, Ruaro JA, Marquetti MGK. Bactericidal effect of high frequency generator in Staphylococcus aureus culture. Fisioter Pesq 2012;19(2):153-7. doi: 10.1590/S1809-29502012000200011

21. Higa DR, Cese PC, Falcão RMM, Cese AC, Chang MR, Borges FS, Madeira-Oliveira JT. Efeito do gerador de alta frequência sobre cultura de candida tropicalis. Rev Esp Fisiot 2007;1(1):1-8

Como citar: SCHUH, Claudia Maria et al. Associação da alta frequência, laser de baixa potência e microcorrentes no tratamento da lesão por pressão. Cinergis, Santa Cruz do Sul, v. 18, n. 2, mar. 2017. ISSN 2177-4005. Disponível em: <https://online.unisc.br/ seer/index.php/cinergis/article/view/8490>. Acesso em: 29 mar. 2017. doi:http://dx.doi.org/10.17058/cinergis.v18i2.8490. 